\title{
Atomic Mechanisms of Gold Nanoparticle Growth in Ionic Liquid by In Situ Scanning Transmission Electron Microscopy
}

\author{
Debora Keller, ${ }^{* a}$ Trond R. Henninen ${ }^{\text {a }}$ and Rolf Erni ${ }^{a}$ \\ a Electron Microscopy Center, Empa - Swiss Federal Laboratories for Materials Science and Technology, Überlandstrasse 129, CH- \\ 8600, Dübendorf, Switzerland. E-mail: debora.keller@empa.ch, rolf.erni@empa.ch.
}

\begin{abstract}
The elementary atomic mechanisms underlying nanoparticle growth in liquids are largely unexplored and mostly subject of conjectures based on theory and indirect experimental insights. Direct, experimental observation of such processes at an atomic level requires imaging with single atom sensitivity and control over kinetics. Although conventional liquid-cell (scanning) transmission electron microscopy ((S)TEM) enables nanoscale studies of dynamic processes, the visualization of atomic processes in the liquid phase is inhibited owing to the liquid film thickness and its encapsulation, both limiting the achievable spatial resolution. In contrast, by using thin, freestanding ionic liquid nanoreactors, this work shows that the mechanisms controlling and triggering particle growth can be uncovered at an atom-by-atom level. Our observations of growing particle ensembles reveal that diverse growth pathways proceed simultaneously. We record Ostwald ripening and oriented particle coalescence tracked at the atomic scale, which confirm the mechanisms suggested by theory. However, we also identify unexpected growth phenomena and more intricate coalescence events which show competing mechanisms. The diversity of the observed growth processes thus illustrates that growth reactions in liquids, on the atomic scale, are much more complex than predicted by theory. Furthermore, this work demonstrates that free-standing ionic liquids enable (sub-)Angström resolution imaging of dynamic processes in liquids with singleatom sensitivity, thus providing a powerful alternative approach to conventional liquid-cell (S)TEM.
\end{abstract}

\section{Introduction}

Tremendous efforts have been dedicated to explore and understand nanoparticle (NP) nucleation and growth dynamics, because fundamental knowledge of initial formation steps is essential for controlling the fabrication process of any specific NP type. NP growth is supposed to proceed either through the classical route of monomer addition and Ostwald ripening ${ }_{1}{ }^{1}$ i.e. small particles dissolve and migrate onto energetically favoured, larger particles, or, alternatively, it proceeds through particle coalescence. Both growth pathways have been extensively studied in the past, theoretically (e.g. ${ }^{2-5}$ ) and experimentally in situ (e.g. $\left.{ }^{6-8}\right)$. However, no solid understanding and control over the growth mechanisms have been attained so far. As both of these fundamental mechanisms can result in comparable particle size distributions, ${ }^{9,10}$ identification of the active growth mechanism requires to track the growth reaction in situ. ${ }^{10}$ Methods like in situ liquid-cell (scanning) transmission electron microscopy (LC-(S)TEM), which provide insight into particle dynamics at the nanoscale, are therefore in high demand to unveil the processes at the early stages of NP growth. However, observing particle growth by monomer attachment, Ostwald ripening or coalescence in situ in a liquid on the atomic scale is still challenging due to the limitations of the spatial resolution in conventional LC-(S)TEM experiments employing SiN electron-transparent windows. ${ }^{11}$ Although atomic resolution has been achieved for dynamics in liquid, the Ostwald ripening process has so far only been captured as an ensemble growth process and not as an atom-by-atom transport mechanism. ${ }^{12-15}$ Likewise, little information is available about particle coalescence studied by 
in situ LC-(S)TEM at atomic resolution. ${ }^{10,16}$ Just recently, Neng et al. reported about a precursor-initiated "jump-to-coalescence" mechanism of gold NPs, recorded by LC-TEM, ${ }^{17}$ where two approaching NPs, below a critical separation distance, suddenly jump and coalescence rapidly. Similar findings were previously reported by others, however, without providing insight into the underlying atomic mechanism. ${ }^{18-}$ ${ }^{20}$ Based on previous work ${ }^{17,21}$, another coalescence mechanism named "bridge-induced contact and fusion" has also been introduced. ${ }^{22}$ There, approaching particles were observed to pause once they reach a critical distance, followed by the formation of a thin atomic bridge between the particles interrupting the hydration layer and enhancing the final coalescence. A special case of coalescence is the oriented attachment. In order to track such processes at atomic resolution in situ, experiments have been done in gas phase ${ }^{23}$ or liquid phase $e^{8,19,24-26}$ in the past. Recently, Zhu et al. presented the oriented attachment of gold NPs observed by LC-TEM where the NPs completely aligned prior to contact. ${ }^{27} \mathrm{~A}$ modified oriented attachment process where particles first get into contact, then successively align and finally fuse into one particle was further observed in vacuum ${ }^{28}$ in environmental (E-)TEM in gas phase, ${ }^{23}$ and in liquid. ${ }^{8}$ These recent studies reveal insights into coalescence processes and possible mechanisms at high spatial resolution, but they mostly focus on entire NPs and only provide little information about the dynamics on an atomic level during the coalescence process.

In this work, we demonstrate that the use of ionic liquids opens a new possibility to increase the resolution in LP-STEM such that the pathway of individual atoms can be monitored during dynamic processes in liquid, e.g. during particle growth. As ionic liquids can be studied in the vacuum environment of the microscope without any encapsulating windows, ${ }^{29,30}$ and because very thin and free-standing liquid layers are feasible, the use of an ionic liquid allows us for improving the spatial resolution in liquid phase experiments. ${ }^{14,31,32} \mathrm{~A}$ resolution of about $1 \AA$ is reached as estimated based on the full width at half maximum of the signal of a single gold atom, ${ }^{33}$ thus, similar resolution as in gas phase can be achieved while still providing a liquid environment. Furthermore, the viscosity of the ionic liquid is affected by the temperature and therefore the speed of the dynamics can be influenced in situ by controlling the temperature. By using a suitable ionic liquid we are able to visualize the motion of individual atoms during particle growth processes, which reveals that, on the atomic scale, much more complex and diverse mechanisms are present than expected from the well-defined, ideal textbook models and from ensemble measurements.

\section{Results and discussion}

The growth of gold NPs in the ionic liquid has been examined at different states, revealing a rich variety of different growth processes. Depending on how the majority of the mass is transported, we suggest a classification of the observed growth mechanisms into three growth types as described in detail in the following subsections: (i) NP growth by attachment of small units (atoms, clusters, ions) that diffuse through the liquid, (ii) rapid particle coalescence, where most of the smaller particle dissolves and its mass is incorporated into the other particle and (iii) slow coalescence, where both particles initially preserve their structure after contact and where both particles are subsequently involved into the orientation, restructuring and fusion process. As indicated by their names, the coalescence processes is typically 
one to two orders of magnitude faster for the rapid coalescence type compared to the slow coalescence type, if compared under the same conditions, i.e. within the same field of view and the same observation period.

\subsection{Nanoparticle growth by monomer attachment and Ostwald ripening}

The addition of single gold atoms or small clusters to existing NPs is the simplest growth process observed. Whereas for small particles $(\leq 2.5 \mathrm{~nm})$ both, monomer attachment and coalescence are active, the addition of single atoms and clusters is the dominant growth pathway for larger particles (> $5 \mathrm{~nm}$ ). Fig. 1 illustrates the growth of a $12 \mathrm{~nm}$ sized particle which proceeds via monomer attachment. The coloured image in Fig. 1a highlights the volume increase of the particle (orange) compared to the initial particle shape (blue) at the beginning of the observation time. Fig. 1b shows the stepwise particle growth over time: A new layer is initiated by an island that is formed in the centre region of the surface and expands over the surface plane ( $86 \mathrm{~s})$. When the new atomic layer is nearly completed, another island is created on top (100 s) and starts growing too (112 s).
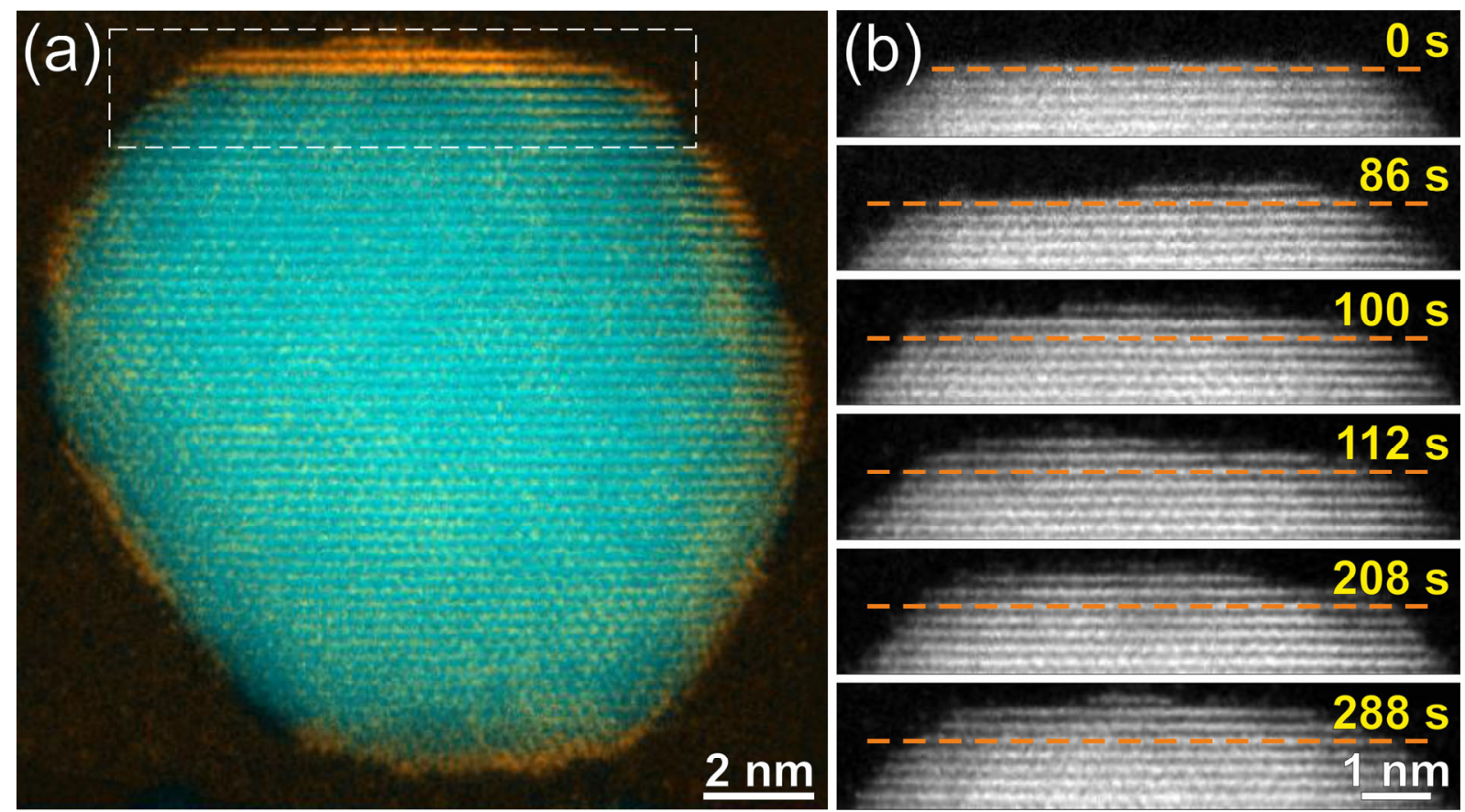

Fig. 1: Particle growth by monomer attachment. In (a), an overlay is shown of the initial particle shape at the beginning of the observation period (blue) with the particle shape $300 \mathrm{~s}$ later (orange). The inset depicts the region, from where in (b) five snapshots taken during the growth process are shown. These snapshots were extracted from Mov. S1 (ESIt).

The gold atoms attached onto the large particle may either stem from precursors present in the ionic liquid that are reduced, or from smaller particles that dissolve in the surrounding of the larger particle, i.e. that undergo Ostwald ripening. Indeed, the typically observed depletion zone of small particles around larger particles (see Fig. S1, ESIt) indicates that Ostwald ripening plays a role in the growth process. In contrast to this indirect confirmation, for smaller particles, direct experimental evidence for Ostwald ripening has frequently been observed. Fig. 2a illustrates two NPs with diameters of about $1.8 \mathrm{~nm}$ and $1.3 \mathrm{~nm}$, that are involved in an Ostwald ripening process. Although some ambiguity remains, we can 
track many single atoms over short periods of time, which provides insights about how mass is transferred from one particle to another. The smaller particle on the right releases single atoms or small clusters (e.g. highlighted by the arrow at $15 \mathrm{~s}$ in Fig. 2a) of which the majority migrates to the neighbouring particle on the left. Whereas shrinkage andgrowth of clearly separated particles has been observed in other examples, the two particles in the present example are initially separated by only a small distance and clear separation between the two particles is not possible throughout the full observation time. Another example (Fig. 2b) shows a combined growth mechanism that includes both classical Ostwald ripening while particles are separated, and also direct mass transport by diffusion along the particles' surface, while the particles are temporarily in contact with each other. Individual atoms migrate through the liquid during the full observation time independent on whether the particles are in contact or not. Yet, as the two particles get in contact at $25 \mathrm{~s}$ (Fig. 2b), most of the smaller particle's remaining mass is transferred to the larger particle at the contact area. Our observations therefore indicate that the atomic scale growth mechanisms are often a combination of different mechanisms that can be neither strictly distinguished, nor attributed to a well-defined, general growth model.
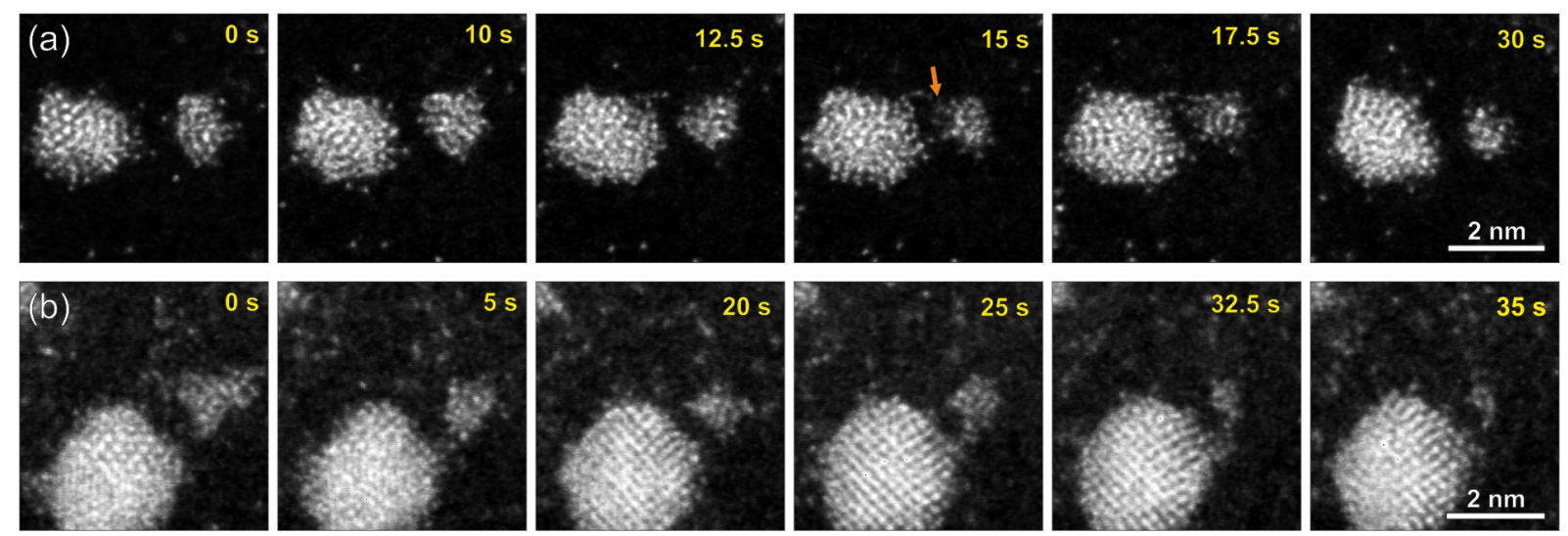

Fig. 2: NP growth by Ostwald ripening. (a) Single atoms and clusters migrate through the ionic liquid from the smaller particle on the right to the larger particle on the left. (b) Two particles are involved in an Ostwald ripening process combined with temporary direct mass transport by surface diffusion (25 - 33 s). The snapshots shown in Fig. $2 a$ and $2 b$ were extracted from Mov. S2a and $2 b(E S /+)$.

\subsection{Rapid coalescence processes}

We have mainly observed rapid coalescence processes for particles smaller than about $1.5 \mathrm{~nm}$. When considering their initiation, coalescence process and end state, there is a large variety of such rapid coalescence processes. Fig. 3a shows an example of a so-called jump-to-coalescence process, ${ }_{1}^{17}$ where a particle merges into another one by a sudden jump. The structure of the smaller, jumping particle collapses and its mass is added to the second particle whose structure is maintained. As highlighted by the arrow $(8 \mathrm{~s})$, the mass of the jumping particle is first added as a protrusion on the opposite side of the larger particle, before it becomes evenly distributed over the particle, which finally relaxes to a spherical shape. This indicates that the smaller particle experiences a sudden, strong acceleration towards the larger particle which makes its mass accumulate first on the opposite side of the larger particle. A similar process has previously been reported by Neng et al. ${ }^{17}$ Based on their first-principles calculations, the presence of precursor monomers in the gap between the two particles can change the energy barrier for coalescence, which then leads to a sudden jump of the two NPs towards each other followed by 
coalescence. As clearly visible in the time series in Fig. 3a, numerous single atoms or precursor molecules are present in the ionic liquid around both particles and also in the gap between them. Therefore, our results support Neng et al.'s explanation regarding the driving force of jump-to-coalescence events and visualize the evolution of this coalescence mechanism in liquid phase at atomic resolution.

Whereas this example of coalescence (Fig. 3a) initiated before the participating particles were in distinct contact with each other, we also observed coalescence events that initiated after a well-defined contact area between the involved particles had been established. As reported by Jin et al. ${ }^{22}$ a small bridge between two particles can lower the activation energy of the coalescence. Whereas only one case has been reported in literature so far by LC-TEM, i.e. the formation of a few atoms wide bridge that initiates the process, ${ }^{22}$ we observed many different starting configurations that can initiate the coalescence process. These range from single atoms and loose atomic structures between the particles (jump-to contact) to distinct single-atomic threads up to contact areas of a full particle surface plane between the particles (bridge-induced coalescence). Two examples of bridge-induced coalescence are shown in Fig. $3 \mathrm{~b}$ and 3c. Fig. $3 \mathrm{~b}$ shows a bridge-induced coalescence with a few atoms wide bridge initiating the process. In the beginning of the observation sequence (at $0 \mathrm{~s}$ ) a two atoms thin bridge between the two particles is formed. Later (at 2-3 s), the bridge broadens and the mass of the smaller particle is gradually transported onto the larger particle via surface diffusion of the atoms. The arrows at $25 \mathrm{~s}$ indicate that also a considerable amount of atoms is transferred to the ionic liquid as single atoms or clusters. After the coalescence process, the larger particle still has a protrusion in the area where the coalescence took place (as indicated by the arrow at $6 \mathrm{~s}$ ) until the particle has fully relaxed into a spherical shape. The other example in Fig. $3 c$ presents a bridge-induced coalescence, where the transferred atoms are added very locally to a facet of the larger particle. After the three atoms wide bridge has been established (after $\sim 6 \mathrm{~s}$ ), single atoms and small clusters migrate from the smaller to the larger particle, where they form two new layers on the surface (at 6.5 and $7 \mathrm{~s}$, indicated by arrows). After completion of these layers ( 10 s), the smaller particle moves slightly upwards to another particle facet and forms a contact area to the larger particle, about 3 to 4 atoms wide. Eventually, most of the smaller particle's mass has merged into the larger particle, while a small fraction of the particle is left in the ionic liquid as clusters and single atoms (25.5 s).

Besides the two shown examples of rapid coalescences induced after the formation of a contact area, we observed a few more examples whereof the size of the initial contact area varied. A further example of such a coalescence event being induced by a larger initial contact area is provided in Fig. S2 (ESIt). Amongst different bridge-induced coalescence events recorded, we observe that the smaller particle migrates towards the larger one and that the bridge is often formed and internupted several times before the coalescence is successful. The collapse of the smaller particle, which triggers the rapid coalescence reaction, could, e.g., be induced by a structural instability of the particle, or by dipole forces due to the relative particle orientation, or also by interruptions of the ionic liquid layers covering the particles surface. However, further investigations are necessary to identify the main cause. In general, such structural collapses are only observed for particles of $1.5 \mathrm{~nm}$ in diameter or less. As it is known that the stability of gold NPs strongly decreases for particles below $5 \mathrm{~nm}$ in size, it seems reasonable that smaller particles 
collapse more frequently. Particles smaller than $1.5 \mathrm{~nm}$ in diameter can even adapt a liquid-like state at room temperature and therefore facilitate structural collapses. ${ }^{34-36}$ In addition to their lower melting temperature, small particles also experience stronger heating due to the energy release after particle contact formation and surface energy reduction. ${ }^{37}$ Based on theory, the coalescence process is expected to proceed faster with an increasing size difference of the participating particles. ${ }^{38}$ In our experiments though, and expectedly under common synthesis conditions, the coalescence speed is dominated by experimental factors such as gold precursor availability, ionic liquid droplet geometry and temperature. Interestingly, we frequently observe that the dissolving smaller particle often leaves single atoms or small sub-nm clusters behind in the ionic liquid (see e.g. Fig. $3 \mathrm{~b}$ and $3 \mathrm{c}$ ), instead of coalescing completely. In addition, the smallest particles are not always involved in a coalescence reaction, but in some examples (see e.g. Fig. 3c) larger particles coalesce while smaller particle nearby do not participate in the reaction. Based on simple thermodynamic considerations, which would ask for a maximum reduction of the surface-to-volume ratio in order to increase particle stability, these findings are surprising and unexpected. Therefore, our results indicate that further factors, e.g. the particle structure, also need to be considered in order to explain such complex particles growth phenomena in liquid phase.
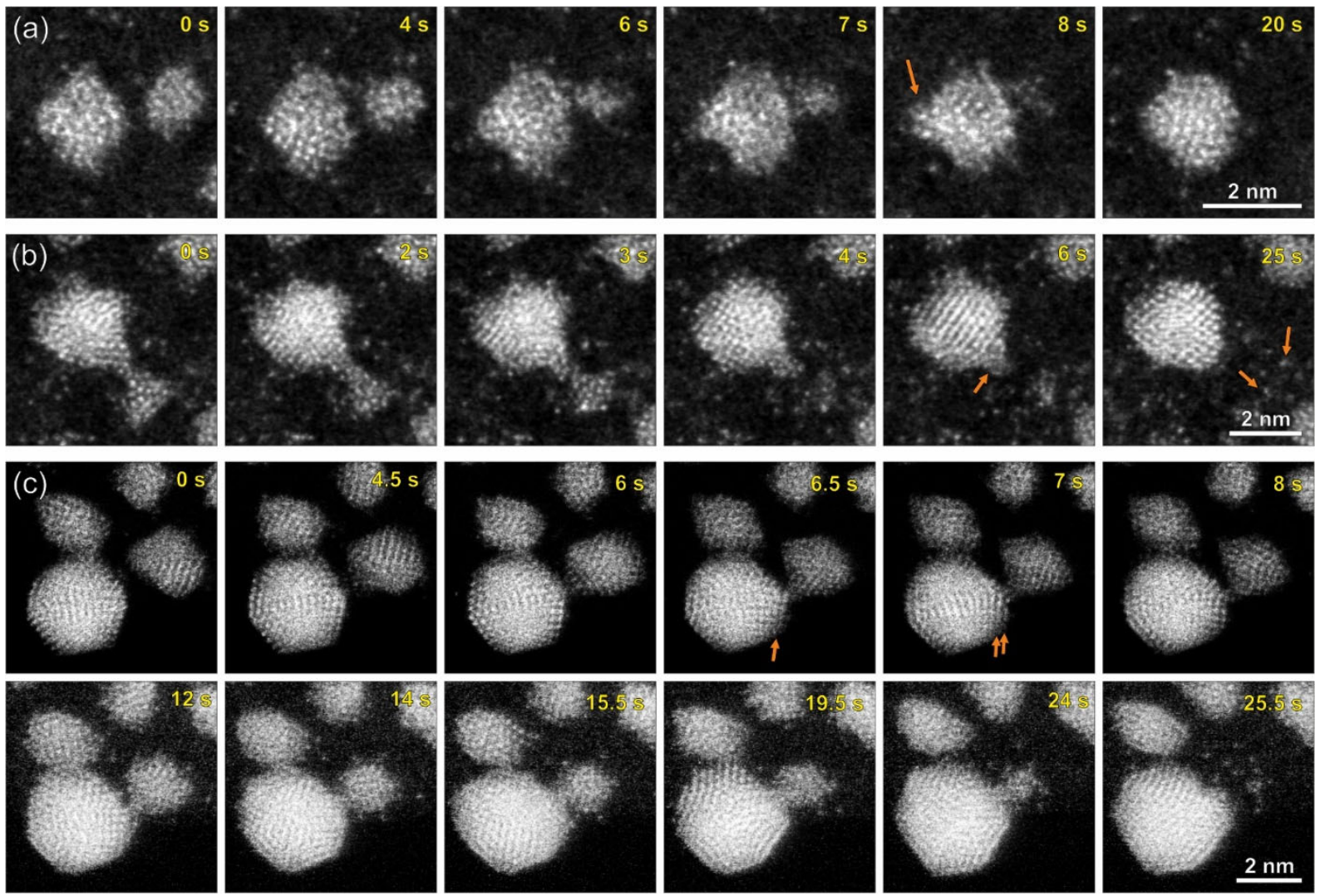

Fig. 3: Rapid coalescence processes. (a) Example of a jump-to-coalescence process of two particles. The arrow indicates the protrusion which is initially formed after coalescence before the particle relaxes to a spherical shape. (b) Example of a bridge-induced coalescence of two particles. The arrow at $6 \mathrm{~s}$ indicates the protrusion formed after coalescence and the arrows at 25 s indicate single atoms which stem from the dissolved particle and remain in the liquid after the coalescence. In (c), an extraordinary example of a bridge-induced coalesce process is shown, where the material transfer from the smaller particle on the right to a specific facet of the larger particle on the left is trackable in detail. The arrows at $6.5 \mathrm{~s}$ and $7 \mathrm{~s}$ show the two layers which are newly formed on the particle facet by the transferred material. The snapshots shown in Fig. 3a, 3b and 3c were extracted from Mov. S3a, S3b and S3c (ESIt). 


\subsection{Slow coalescence through particle orientation and restructuring}

In contrast to the rapid coalescences shown above, we also recorded slower coalescence processes, typically for NPs larger than about $1 \mathrm{~nm}$ (i.e. which are more stable), but smaller than about $2.5 \mathrm{~nm}$ and thus mobile enough to undergo coalescence. ${ }^{37}$ In this case, none of the particles collapses but both of them follow a slow reorientation and restructuring process until appropriate crystal alignment in respect to each other is reached. Thereafter, they merge into a single nanocrystal. Fig. 4 illustrates an example of two small particles that merge by such a slow coalescence. In contrast to the typical oriented attachment process reported in the literature (e.g. ${ }^{39,40}$ ) where particle alignment happens prior to contact, the particles in our system only start orienting themselves after having formed a common contact area. Once alignment is reached, the particles fuse via neck formation and diffusion. A similar process to what we describe here has been observed earlier in another ionic liquid system. ${ }^{14}$ As the behaviour of ionic liquids as solvents is different from the behaviour of water, the specific properties of the ionic liquid might prevent a contact-free particle alignment. The charges inherent to the ionic liquids might distort or shield the local Coulomb interactions and the short-term dipole forces between the particles, which are necessary for contact-free particle alignment. ${ }^{41}$ An additional example of a slow coalescence process, where four particles slowly coalesce, is provided in Fig. S3 (ESI+) and Mov. S4b (ESI+).
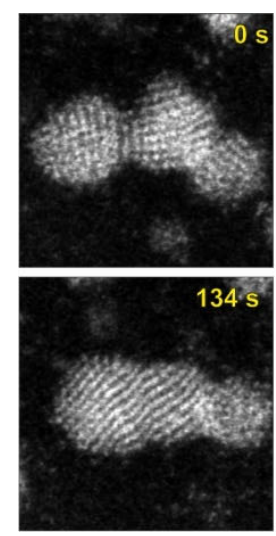
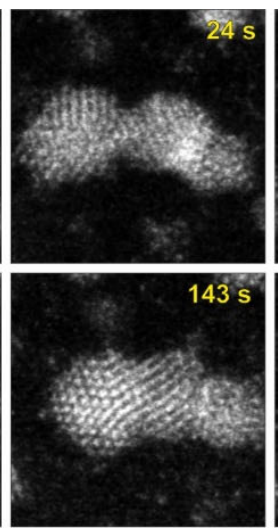
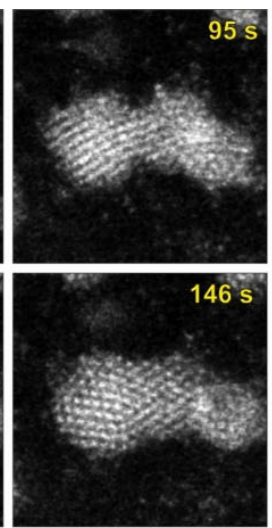
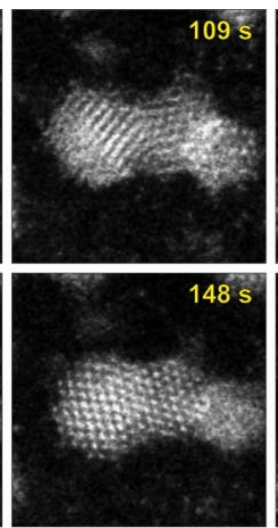
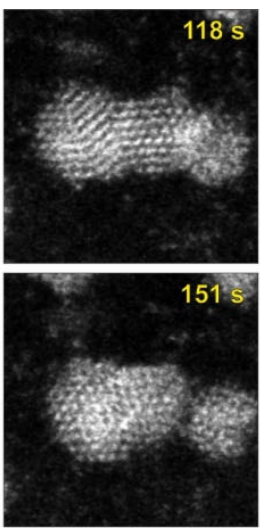
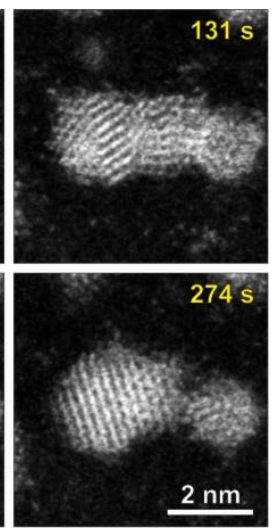

Fig. 4: Slow coalescence process. Two particles coalesce, proceeding via contact formation, alignment and restructuring of both particles, and finally particle fusion. The snapshots shown in Fig. 4 were extracted from Mov. S4a (ESI)).

\section{Conclusions}

In this work, we study gold NP growth in a liquid environment by in situ STEM at atomic resolution. Using an ionic liquid as liquid phase instead of a liquid cell allows us for increasing the resolution to about $1 \AA$ with single-atom sensitivity. This enables the observation and tracking of individual atoms involved in NP growth processes. The insights gained by this method depict the atom-by-atom evolution of the particles involved in different growth processes such as particle growth by monomer attachment and Ostwald ripening as well as different types of particle coalescence events. We observe that various NP growth trajectories are simultaneously active in the same liquid droplet and that different mass transport pathways can even act in parallel on the same particle. An example of such a combined growth process is illustrated in the case of an Ostwald ripening event that is monitored in detail. Amongst the recorded coalescence events, 
we distinguish between fast and slow coalescence processes. Very small particles typically undergo rapid coalescence processes, which are correlated with a sudden collapse of the smaller particle's structure and the subsequent fusion of the involved particles. Furthermore, our method allows for visualizing the recently described jump-to-coalescence and bridge-induced coalescence mechanisms on an atomic level, thus confirming the mechanisms previously suggested. For particles of intermediate size, whose thermodynamic stability must be more favourable compared to the smaller particles, we typically observe slow coalescence processes. In the presented examples, we can track the subsequent steps of contact formation, reorientation, restructuring and finally particle fusion on the atomic scale. Whereas particle coalescence only takes place for small particles (particles $<1.5 \mathrm{~nm}$ for rapid coalescence) and intermediate sized particles (1.0 $2.5 \mathrm{~nm}$ for slow coalescences), the growth by Ostwald ripening and monomer attachment occurs also for larger particles. Thus, our observations clearly show that the initial particle growth is critically affected by the particle stability based on the surface-to-volume ratio. However, other findings such as sub-nm clusters left behind in the liquid after a coalescence event, are unexpected from basic thermodynamic considerations.

In summary, our work demonstrates the potential of free-standing ionic liquids for accessing dynamic, atomic-scale processes in situ as an alternative approach to conventional LC-STEM. The improvement in spatial resolution opens new possibilities for accessing dynamic processes with single-atom sensitivity in liquid phase by STEM. The impact of the enhanced resolution is highlighted in our study of Au particle growth processes which can be tracked in detail by this method. Our recordings present clear, experimental insights into different NP growth processes. While some examples experimentally confirm previously reported mechanisms that were only described in theory, other examples reveal more complex and unexpected particle growth processes. Therefore, due to the improved spatial resolution of this new in-situ method, our observations allow us to show the diversity, complexity and dynamics of particle growth processes in liquids.

\section{Experimental}

\subsection{Materials and TEM sample preparation}

The ionic liquid tetrabutylammonium chloride $\left(\left[\mathrm{Bu}_{4} \mathrm{~N}\right][\mathrm{Cl}]\right.$, purity $\left.\geq 97.0 \%\right)$, the gold precursor sodium tetrachloroaurate(III) dihydrate $\left(\mathrm{NaAuCl}_{4} \cdot 2 \mathrm{H}_{2} \mathrm{O}\right.$, purity $\left.\geq 99.0 \%\right)$ and oxalic acid $\left(\mathrm{C}_{2} \mathrm{H}_{2} \mathrm{O}_{4}\right.$, purity $\geq 99.0 \%$ ) purchased from Sigma Aldrich were used as received. Firstly, samples containing a low concentration of gold $(10 \mathrm{mM})$ in the ionic liquid were prepared which allow for observing few gold particles in the liquid. Although the estimated melting temperature of bulk $\left[\mathrm{Bu}_{4} \mathrm{~N}\right][\mathrm{Cl}]$ is $70^{\circ} \mathrm{C}$, when used in small quantities, this ionic liquid appears to be viscous already at room temperature. Nevertheless, $\mathrm{NaAuCl}_{4} \cdot 2 \mathrm{H}_{2} \mathrm{O}$ and $\left[\mathrm{Bu}_{4} \mathrm{~N}\right][\mathrm{Cl}]$ were dissolved in methanol, at a volume ratio of $\left[\mathrm{Bu}_{4} \mathrm{~N}\right][\mathrm{Cl}]$ :methanol of $\sim 1: 700$, in order to manipulate small amounts of the solution as described elsewhere. ${ }^{32}$ Thus various nanoreactors, i.e. different small ionic liquid droplets containing gold precursor, were deposited on the heating E-chips from Protochips. It is known that 
radicals of $\left[\mathrm{Bu}_{4} \mathrm{~N}\right][\mathrm{Cl}]$ are less stable than radicals of other solvents or other ionic liquids containing aromatic cations. Therefore, solvated electrons that are introduced by the electron beam can survive over long times in $\left[\mathrm{Bu}_{4} \mathrm{~N}\right][\mathrm{Cl}]$ and can react with the sample. ${ }^{42-45}$ In solutions containing only small amounts of gold precursor, we expect that solvated electrons can reduce some of the gold ions to neutral gold atoms under electron irradiation. Due to the stabilizing characteristics of ionic liquids (see e.g. ${ }^{29,46,47}$, no surfactant is needed but $\left[\mathrm{Bu}_{4} \mathrm{~N}\right][\mathrm{Cl}]$ is expected to act as a stabilizing agent for formed gold particles.

Secondly, samples with much higher gold concentration (150 mM) leading to higher particle densities in the liquid were prepared. This concentration is comparable to concentrations used in previous studies, ${ }^{14,30}$ and it allowed us for observing a multitude of particles growing simultaneously within small fields of view. In order to reduce such high concentrations of gold ions in $\left[\mathrm{Bu}_{4} \mathrm{~N}\right][\mathrm{Cl}]$, oxalic acid was further added to these samples. The oxalic acid was dissolved in ethanol $(10 \mathrm{mM})$ and similarly added to the E-chip as the ionic liquid. Methanol and ethanol are expected to evaporate prior to the experiment, but it cannot be excluded that small amounts of these solvents remain as bubbles in the ionic liquid and cause contaminations during the experiments. The results shown in Fig. 3c and S3 were obtained using low gold concentrations whereas the other results were recorded on samples containing higher gold concentrations and oxalic acid.

\subsection{In situ STEM experiments}

All experiments were performed using a Protochips Fusion 500 heating holder and an FEI Titan Themis TEM/STEM equipped with a probe Cs corrector, operated at $300 \mathrm{kV}$. The thickness of the liquid was roughly estimated by the liquid contrast relative to the contrast of the carbon film (of about 15-20 nm). For the results shown in this work, only areas of liquid layers of approximately $50 \mathrm{~nm}$ or less were studied, as atomic resolution is hard to achieve in thicker layers. A more detailed description of the ionic liquid thickness is provided in the ESIt.

By in situ heating, the viscosity of the liquid could be changed in order to accelerate or slow down the reactions of interest in situ. Experiments were performed either at room temperature (see Fig. $3 \mathrm{c}$ and 4, Fig. S1, S2 and S3 (ESIt), or at elevated temperatures of $40^{\circ} \mathrm{C}$ (see Fig. 2a, 2b and $3 \mathrm{~b}$ ) or $100{ }^{\circ} \mathrm{C}$ (see Fig. 1 and 3a). Amongst all experiments performed, we did not find a dependency of the particle growth type on the temperature, but the kinetics could clearly be changed by heating. Therefore, despite the fact that kinetics have been varied between different experiments, we observed reproducibly that different growth processes take place simultaneously within all the investigated nanoreactors. We expect that most of the observed particles are not in contact with the carbon foil, as gold particles are typically present throughout the whole focal depth of the ionic liquid droplet. Due to the high magnification necessary for dynamic atomic resolution imaging, the dose rates were in the range of $2-8.5^{\star} 10^{\wedge} 4 \mathrm{e}^{-} \mathrm{A}^{-2} \mathrm{~s}^{-1}$, similar to dose 
rates previously reported in similar experiments. ${ }^{31,48} \mathrm{As}\left[\mathrm{Bu}_{4} \mathrm{~N}\right][\mathrm{Cl}]$ is very beam-resistant compared to other imidazolium-based ionic liquids and aqueous solutions, ${ }^{42-45}$ it is expected to withstand electron irradiation of the applied dose. Indeed, the dose rates and total dose used in our experiments did not lead to any visible degradation of the ionic liquid. The specific dose rates and further acquisition parameters used for the presented figures and movies are provided in Tab. S1 (ESI+). A more detailed discussion of possible beam effects is also provided in the Supplementary Information (ESI+).

\subsection{Data processing}

The time series recorded were analysed and further processed using DigitalMicrograph ${ }^{\mathrm{TM}}$ and Fiji. To record the rapid coalescence dynamics, the data was recorded at a high frame rate, giving noisy raw data. For Fig. 1, only a simple median filter was applied due to a high signal in the raw data. The rest of the displayed figures and movies were denoised using PGURE-SVT, which applies spatial and temporal correlation to distinguish between signal and noise. ${ }^{49}$ Due to high background signal from the ionic liquid in Fig. S2 (ESI+) and Mov. S3d (ESI+) we also applied a peak-of-histogram background subtraction for this movie..$^{50}$

\section{Conflicts of interest}

There are no conflicts to declare.

\section{Acknowledgement}

This work has received funding from the European Research Council (ERC) under the EU's Horizon 2020 research and innovation program (grant agreement No. 681312).

\section{References}

1 W. Ostwald, Z. Phys. Chem., 1896, 22, 289-330.

2 I. M. Lifshitz and V. V. Slyozov, J. Phys. Chem. Solids, 1961, 19, 35-50.

3 C. Wagner, Ber. Bunsenges. Phys. Chem., 1961, 65, 581-591.

4 A. Baldan, J. Mater. Sci., 2002, 37, 2171-2202.

5 N. T. K. Thanh, N. Maclean and S. Mahiddine, Chem. Rev., 2014, 114, 7610-7630.

6 A. De Clercq, W. Dachraoui, O. Margeat, K. Pelzer, C. R. Henry and S. Giorgio, J. Phys. Chem. Lett., 2014, 5, $2126-2130$.

7 T. J. Woehl, J. E. Evans, I. Arslan, W. D. Ristenpart and N. D. Browning, ACS Nano, 2012, 6, 8599-8610.

8 H.-G. Liao, L. Cui, S. Whitelam and H. Zheng, Science, 2012, 336, 1011-1014.

9 H. Zheng, R. K. Smith, Y. Jun, C. Kisielowski, U. Dahmen and A. P. Alivisatos, Science, 2009, 324, 1309-1312.

10 J. Polte, CrystEngComm, 2015, 17, 6809-6830.

11 F. M. Ross, Science, 2015, 350, aaa9886.

12 D. Alloyeau, T. Oikawa, J. Nelayah, G. Wang and C. Ricolleau, Appl. Phys. Lett., 2012, $101,121920$.

13 T. E. Martin, P. L. Gai and E. D. Boyes, Microsc. Microanal., 2015, 7, 3705-3711.

14 T. Uematsu, M. Baba, Y. Oshima, T. Tsuda, T. Torimoto and S. Kuwabata, J. Am. Chem.Soc., 2014, 136, $13789-13797$. 
H. Zhang, J. J. De Yoreo and J. F. Banfield, ACS Nano, 2014, 8, 6526-6530.

W. Neng, L. Shuang-ying, X. Jun and M. Martini, Nanotechnology, 2016, 27, 205605.

U. Anand, J. Lu, D. Loh, Z. Aabdin and U. Mirsaidov, Nano Lett., 2016, 16, 786-790.

D. Li, M. H. Nielsen, J. R. I. Lee, C. Frandsen, J. F. Banfield and J. J. D. Yoreo, Science, 2012, 336, 1014-1018.

X. Zhang, Y. He, M. L. Sushko, J. Liu, L. Luo, J. J. D. Yoreo, S. X. Mao, C. Wang and K. M. Rosso, Science, 2017, 356, 434437.

21 P. Schapotschnikow, M. A. van Huis, H. W. Zandbergen, D. Vanmaekelbergh and T. J. H. Vlugt, Nano Lett., 2010, 10, 3966-3971.

22 B. Jin, M. L. Sushko, Z. Liu, C. Jin and R. Tang, Nano Lett., 2018, 18, 6551-6556.

23 M. A. van Huis, L. T. Kunneman, K. Overgaag, Q. Xu, G. Pandraud, H. W. Zandbergen and D. Vanmaekelbergh, Nano Lett., 2008, 8, 3959-3963.

24 J. M. Yuk, J. Park, P. Ercius, K. Kim, D. J. Hellebusch, M. F. Crommie, J. Y. Lee, A. Zettl and A. P. Alivisatos, Science, 2012, $336,61-64$.

25 Z. Aabdin, J. Lu, X. Zhu, U. Anand, N. D. Loh, H. Su and U. Mirsaidov, Nano Lett., 2014, 14, 6639-6643.

26 W. Wei, H. Zhang, W. Wang, M. Dong, M. Nie, L. Sun and F. Xu, ACS Appl. Mater. Interfaces, 2019, 11, $24478-24484$.

27 C. Zhu, S. Liang, E. Song, Y. Zhou, W. Wang, F. Shan, Y. Shi, C. Hao, K. Yin, T. Zhang, J. Liu, H. Zheng and L. Sun, Nat. Commun., 2018, 9, 421.

28 J. M. Yuk, M. Jeong, S. Y. Kim, H. K. Seo, J. Kim and J. Y. Lee, Chem. Commun., 2013, 49, 11479-11481.

29 C. Janiak, in Ionic Liquids (ILs) in Organometallic Catalysis, eds. J. Dupont and L. Kollár, Springer Berlin Heidelberg, Berlin, Heidelberg, 2015, pp. 17-53.

30 A. Imanishi, S. Gonsui, T. Tsuda, S. Kuwabata and K. Fukui, Phys. Chem. Chem. Phys., 2011, 13, 14823-14830.

31 T. Miyata, F. Uesugi and T. Mizoguchi, Sci. Adv., 2017, 3, e1701546.

32 D. Keller, T. R. Henninen and R. Erni, Micron, 2019, 117, 16-21.

33 P. E. Batson, N. Dellby and O. L. Krivanek, Nature, 2002, 418, 617-620.

34 Ph. Buffat and J.-P. Borel, Phys. Rev. A, 1976, 13, 2287-2298.

35 G. Schmid and B. Corain, Eur. J. Inorg. Chem., 2003, 2003, 3081-3098.

36 D. Knez, M. Schnedlitz, M. Lasserus, A. Schiffmann, W. E. Ernst and F. Hofer, Ultramicroscopy, 2018, 192, 69-79.

37 M. José-Yacamán, C. Gutierrez-Wing, M. Miki, D.-Q. Yang, K. N. Piyakis and E. Sacher, J. Phys. Chem. B, 2005, 109, 97039711.

38 T. Hawa and M. R. Zachariah, J. Aerosol Sci., 2006, 37, 1-15.

39 B. H. Kim, J. Yang, D. Lee, B. K. Choi, T. Hyeon and J. Park, Adv. Mater., 2018, 30, 1703316.

40 W. Lv, W. He, X. Wang, Y. Niu, H. Cao, J. H. Dickerson and Z. Wang, Nanoscale, 2014, 6, 2531-2547.

41 H. Zhang and J. F. Banfield, CrystEngComm, 2014, 16, 1568-1578.

42 É. Bossé, L. Berthon, N. Zorz, J. Monget, C. Berthon, I. Bisel, S. Legand and P. Moisy, Dalton Trans., 2008, 924-931.

43 E. M. Siedlecka, M. Czerwicka and S. S. and P. Stepnowski, Curr. Org. Chem., 2011, 15, 1974-1991.

44 J. F. Wishart and P. Neta, J. Phys. Chem. B, 2003, 107, 7261-7267.

45 T. Tsuda, S. Seino and S. Kuwabata, Chem. Commun., 2009, 0, 6792-6794.

46 I. Capek, in Noble Metal Nanoparticles: Preparation, Composite Nanostructures, Biodecoration and Collective Properties, ed. I. Capek, Springer Japan, Tokyo, 2017, pp. 211-316.

47 M. Farren-Dai, E. Awoonor-Williams, C. S. MacNeil, Z. Mahimwalla and K. Ghandi, Chemical Physics Letters, 2014, 610611, 331-334.

48 T. R. Henninen, M. Bon, F. Wang, D. Passerone and R. Erni, Angewandte Chemie, 2020, 132, 849-855.

49 T. Furnival, R. K. Leary and P. A. Midgley, Ultramicroscopy, 2017, 178, 112-124. 
50 T. R. Henninen, Peak-of-histogram, https://github.com/hentr/peak-of-histogram, (accessed October 30, 2019). 